Gynäkologische Endokrinologie 2010 · 8:5-6 DOI 10.1007/s10304-009-0334-5

Online publiziert: 29. Januar 2010

(c) Springer-Verlag 2010

\author{
R. Felberbaum ${ }^{1} \cdot$ K. Diedrich ${ }^{2} \cdot 0$. Ortmann $^{3} \cdot$ W. Küpker ${ }^{4}$ \\ ${ }^{1}$ Klinik für Frauenheilkunde und Geburtshilfe, Perinatalzentrum, Zentrum \\ für Reproduktionsmedizin, Klinikum Kempten - Oberallgäu gGmbH, Kempten \\ ${ }^{2}$ Klinik für Frauenheilkunde und Geburtshilfe, Universitätsklinikum \\ Schleswig-Holstein, Campus Lübeck, Lübeck \\ ${ }^{3}$ Universitätsfrauenklinik Regensburg, Regensburg \\ ${ }^{4}$ Endometriosezentrum Baden-Baden, Baden-Baden
}

\title{
Gynäkologische Onkoendokrinologie
}

zutage ohne endokrine Behandlungsformen nicht mehr denkbar. Bis 2005 war dabei der Östrogenantagonist Tamoxifen der Goldstandard in der adjuvanten Therapie. In großen Phase-III-Studien konnte inzwischen gezeigt werden, dass die Aromataseinhibitoren der alleinigen Tamoxifentherapie in der Postmenopause überlegen sind.

Für prämenopausale Patientinnen mit gynäkologischen Malignomen stellt die medikamentöse oder operative Kastration eine schwere Belastung dar. Die schwerwiegendsten Folgen des Östrogenmangels sind Hitzewallungen, Nachtschweiß, urogenitale Atrophie, Osteoporose und möglicherweise auch ein erhöhtes Risiko für kardiovaskuläre Erkrankungen. Die Vorstellung, diesen Patientinnen durch eine Hormonersatztherapie zu einer besseren Lebensqualität $\mathrm{zu}$ verhelfen, liegt also nahe. Leider kann in solchen Fällen eine schädigende Wirkung nie ausgeschlossen werden. Sowohl für das Mamma- als auch für das Ovarialkarzinom wären große prospektive Studien zu dieser klinisch sehr relevanten Fragestellung wünschenswert.

Auch wenn die gynäkologische Onkoendokrinologie sich abseits von Fragen der Fertilität und der Kinderwunschbehandlung entwickelt hat, so gewinnt der Problemkreis der Fertilitätsprotektion unter onkologischer Therapie in den letz- ten Jahren immer mehr an Bedeutung. Es ist die verbesserte Therapie von Patientinnen, z. B. mit Mammakarzinom, die generell zu einem verbesserten Gesamtüberleben geführt hat. Dies führt dazu, dass immer mehr junge Frauen nach überstandener onkologischer Therapie über die Möglichkeiten einer Schwangerschaft und der Geburt eines Kindes nachdenken. Leider erfüllen sich nicht alle Hoffnungen in neue Therapieansätze. So scheinen GnRH-Analoga nicht vor einem vorzeitigen Erlöschen der Ovarialfunktion bei Patientinnen mit Mammakarzinom und wohl auch nicht bei anderen Malignomen zu schützen.

In der näheren Zukunft werden neue, hormonassoziierte Therapieformen unsere Behandlungsmöglichkeiten erweitern. Die rezeptorvermittelte „targeted therapy“, z. B. mit GnRH- oder GHRHAntagonisten und daran gekoppelten zytotoxischen Agenzien hat sich in präklinischen Tumormodellen des Mammakarzinoms bei geringer Nebenwirkungstoxizität als hoch wirksam erwiesen. Erste Phase-II-Studien werden nun mit diesen Hybridpeptidhormonen durchgeführt.

In dem vorliegenden Heft haben wir versucht, das gesamte Spektrum der gynäkologischen Onkoendokrinologie auszuleuchten. Es ist uns gelungen, für die Beiträge ausgewiesene Experten für
Gerade die Therapie des hormonresponsiven Mammakarzinoms ist heut- 
die jeweiligen Themenkomplexe zu gewinnen. Dafür gilt allen Autoren unser Dank. Es ist ihnen gelungen, die verschiedenen Facetten des Themas im Spannungsfeld zwischen Risikoerhöhung und Protektion zu beschreiben.

Und dabei wird klar: „Die gesamte Frauenheilkunde ist Endokrinologie!“

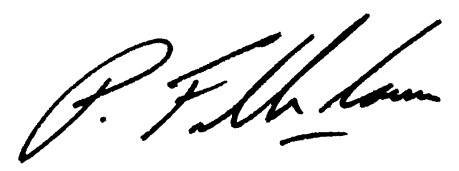

Ricardo Felberbaum

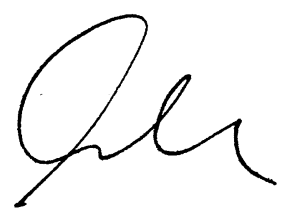

Klaus Diedrich

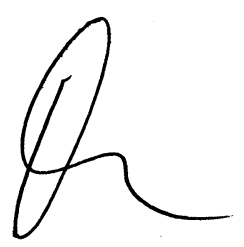

Olaf Ortmann

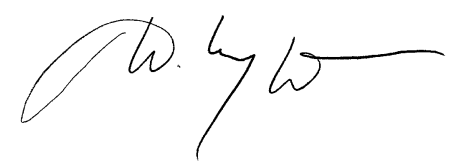

Wolfgang Küpker

\section{Korrespondenzadresse}

\section{Prof. Dr. R. Felberbaum}

Klinik für Frauenheilkunde und Geburtshilfe, Perinatalzentrum, Zentrum für Reproduktionsmedizin, Klinikum Kempten - Oberallgäu gGmbH Robert-Weixler-Str. 50, 87439 Kempten Ricardo.Felberbaum@Klinikum-Kempten.de

\section{Themenübersicht}

Die „Gynäkologische Endokrinologie“ bietet Ihnen umfassende und aktuelle Beiträge zu interessanten Themenschwerpunkten aus allen Bereichen der gynäkologischen Endokrinologie und Reproduktionsmedizin.

Wir haben die Jahrgänge 2009/2010 im Überblick für Sie zusammengestellt:

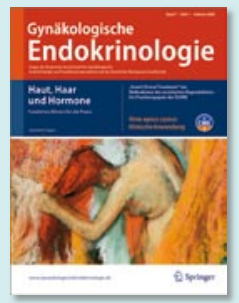

\section{9}

- Heft 1/09 Haut, Haar und Hormone

- Heft 2/09 Assistierte Reproduktion: Bessere Ergebnisse durch ergänzende Therapieoptionen?

- Heft 3/09 Störungen der Geschlechtsidentität

- Heft 4/09 Schilddrüse

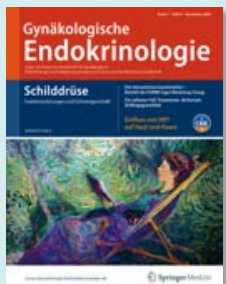

2010

- Heft 1/10 Onkologische Endokrinologie

- Heft 2/10 Mythen in der Reproduktionsmedizin

- Heft 3/10 Ovar: von der Fetalperiode bis zur Menopause

- Heft 4/10 Endokrinologie, Stress und Leistungssport

Bestellen Sie einzelne Ausgaben oder abonnieren Sie die Zeitschrift zum Preis von EUR 119,- pro Jahr (zzgl. Versandkosten, Ermäßigung für Ärzte in Aus- und Weiterbildung)! Unser Kundenservice steht Ihnen für Fragen und Informationen gerne zur Verfügung:

Springer-Verlag, Kundenservice Zeitschriften

Haberstr. 7, 69126 Heidelberg

Tel.: +49 6221-345-4303, Fax: +49 6221-345-4229

E-Mail: leserservice@springer.com

\section{Beiträge auf einen Blick}

Abonennten haben online Zugriff auf alle Beiträge im elektronischen Volltextarchiv unter www.GynaekologischeEndokrinologie.de.

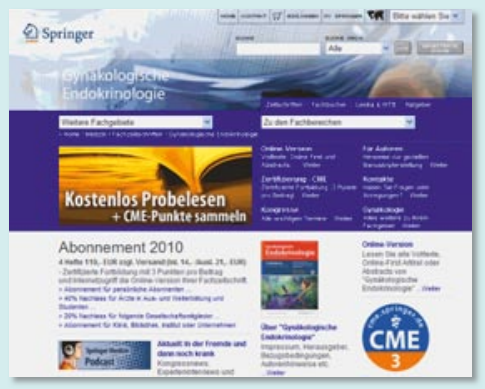

Eine interessante Lektüre wünscht Ihnen

Ihre Redaktion

Fachzeitschriften Medizin /Psychologie
Ins Volltextarchiv gelangen Sie von der Startseite über den Navigationspunkt "Online Version".

Nach einer einmaligen Registrierung können Sie die Beiträge als pdf oder in der html-Version abrufen. 